\title{
On the location of a neck formation during the tension of cylindrical specimens
}

\author{
E. Yu. Lubkova, E. M. Morozov, A. V. Osintsev ${ }^{\dagger}$, A. S. Plotnikov \\ †AVOsintsev@yandex.ru \\ National research nuclear university “MEPhI”, Kashirskoe shosse 31, 115409, Moscow, Russia
}

\begin{abstract}
Numerical simulations of the neck formation during tension of cylindrical specimens have been carried out in an approach of simplified elastoplastic properties of the material and large strains. The results have been compared to those of a similar simulation done in another computation code. Regularities of the strain localization and neck development, dependence of the neck location on elastoplastic properties of material, in particular, on strain hardening modulus are illustrated. Tensile tests of cylindrical samples of different types and sizes have been carried out. It has been shown that in the samples of the same type and sizes made of the same material, the neck is formed dominantly on the same place. Strain diagrams of materials obtained in tests are transformed into true stress - strain diagrams. These diagrams have been used as models of the elastoplastic behavior of materials in simulations of the neck formation on models imitating the experimental samples. A qualitative agreement of the test and numerical simulation results have been obtained. In simulations of the neck formation, in some cases the formation of a pair of symmetrically located necks have been found. This prediction was verified in tensile stress experiments with real time registration of deformation by the method of digital image correlation. It has been found that following the stage of uniform straining, two symmetrically located regions of concentration of transverse strains are formed. Eventually one of these regions breaks up, while the second one gives rise to a neck, which in turn results in the failure of the sample.
\end{abstract}

Keywords: finite element method, digital image correlation method, tensile cylindrical specimens, necking.

\section{К вопросу о месте образования шейки при растяжении цилиндрических образцов}

\author{
Лубкова Е. Ю., Морозов Е.М., Осинцев А.В. ${ }^{\dagger}$, Плотников А. С.
}

Национальный исследовательский ядерный университет «МИФИ», Каширское шоссе 31, 115409, Москва, Россия

Проведено численное моделирование процесса образования шейки при растяжении цилиндрического образца с учётом упрощённых упругопластических свойств материала и больших деформаций. Результаты сопоставлены с имеющейся публикацией аналогичного моделирования в другом расчётном коде. Проиллюстрированы закономерности локализации и развития шейки, зависимость положения шейки от упругопластических свойств материала, в частности - модуля упрочнения. Проведены испытания цилиндрических образцов различного типоразмера на одноосное растяжение. Показано, что в образцах одного типоразмера, изготовленных из одного материала, шейка локализуется преимущественно в одном и том же месте. Диаграммы деформирования материалов, полученные в результате испытаний, переведены в диаграммы истинных напряжений и истинных деформаций. Данные диаграммы были использованы в качестве модели упругопластического поведения материалов при проведении моделирования процесса образования шейки при растяжении на моделях, повторяющих экспериментальные образцы. В результате моделирования получено качественное совпадение результатов испытаний с результатами численного моделирования. При численном моделировании процесса образования шейки в некоторых случаях наблюдалось образование двух симметрично расположенных шеек в одной модели. Для проверки этого факта было проведено испытание образца на растяжение с регистрацией деформаций на поверхности образца в реальном масштабе времени методом корреляции цифровых изображений. Установлено, что после стадии равномерных деформаций образуются две симметрично расположенные области концентрации поперечных деформаций, одна из которых в процессе дальнейшего растяжения расформировывается, а на месте второй образовывается шейка, приводящая в конечном итоге к разрыву.

Ключевые слова: метод конечных элементов, метод цифровой корреляции изображений, растяжение цилиндрических образцов, образование шейки. 


\section{1. Введение}

Современные требования к расчётному обоснованию прочностной надёжности конструкций требуют проведения анализа, способов практического выполнения которого раньше попросту не существовало. Так, необходимость обеспечения прочности на предельных и запредельных режимах работы или аварийных процессах, обязывает учитывать такие особенности поведения материала, как развитая пластическая деформация, большие деформации, разрушение, а также их динамику.

Технически, современные расчётные методики, такие как метод конечных элементов, метод сглаженных частиц и другие, готовы к реализации учёта подобных эффектов. Однако одновременно неизбежно встаёт вопрос верификации подобных результатов.

Известно, что при испытании на растяжение до разрушения, большинство цилиндрических образцов из пластичных материалов перед самым моментом разрушения заметно изменяют форму. Подобное явление резкого утонения образца, локализованного на месте последующего разрушения, называется образованием шейки.

Образование шейки связано с переходом от равномерной пластической деформации по всей длине образца к неравномерной, локальной деформации. Деформация концентрируется и быстро растет в одном месте. Принято считать, что процесс образования шейки обусловлен проявлением неустойчивости цилиндрической формы стержня при его растяжении. Момент потери устойчивости на условной кривой деформирования совпадает с точкой максимума, которую называют точкой бифуркации [1].

Соответствующие механические характеристики предел прочности (временное сопротивление) и равномерная деформация по Я.Б. Фридману называются критическими [2]. В теории прочности, в случае идеальной однородности материала и геометрии, место расположения шейки принято считать произвольным (случайным), т.е. точно предсказать, в каком сечении образец порвется, человек не в состоянии. В реальной обстановке естественно ожидать, что шейка образуется на месте начальной неоднородности или возможной концентрации напряжений.

После потери устойчивости, напряженно-деформированное состояние в области шейки становится сложным и неоднородным. Помимо продольных напряжений в круглых образцах возникают радиальные и окружные напряжения и деформации, которые начинают существенно влиять на процесс деформирования. Исследования после потери устойчивости проводились Н. Н. Давиденковым [3], А. М. Жуковым [4] и В.Г. Кутяйкиным [5] на предмет распределения напряжений и деформаций вдоль радиуса в наименьшем сечении шейки.

Данный эффект, достаточно сложный для численного описания, может быть получен в весьма простом эксперименте. Представляется разумным использовать его в качестве верификационного для сопоставления с результатами численного моделирования.

\section{2. Моделирование процесса образования шейки Д. В. Бережновым и В.Н. Паймушиным}

В работе [6] исследованы две постановки упругопластических задач механики деформируемых твердых тел при конечных перемещениях и деформациях. Первая постановка за меру деформаций принимает компоненты тензора деформаций Коши-Грина, связанные с компонентами тензора условных напряжений физически нелинейными соотношениями по теории течения. Вторая постановка основана на введении истинных деформаций удлинений и сдвигов, связанных с компонентами истинных напряжений по В. В. Новожилову [7].

Условие текучести и связь между приращениями компонент тензора напряжений и тензора деформаций в первом варианте записываются в обобщенных напряжениях и с помощью уравнений Прандтля-Рейса. Во втором варианте условие текучести и уравнения ПрандтляРейса записываются с помощью компонент истинных напряжений и деформаций.

Расчёт производился при помощи разработанного авторами расчётного конечно-элементного кода. В качестве модели (рис. 1) использовалась $1 / 4$ часть цилиндрического образца длиной и диаметром рабочей части 200 мм и 10 мм соответственно, длиной и диаметром головок 20 мм и 16 мм соответственно и радиусом галтели 3 мм. Граничные условия модели представляли собой $u=0$ и $v=0$ на участках $\mathrm{DE}$ и $\mathrm{EF}$ соответственно, где $u$ - продольные, а $v$ - поперечные перемещения образца. Нагружение проводилось во всех точках участка BC контура образца, пошагово задавая перемещение $U$. Расчёт производится в осесимметричной постановке. Конечно-элементная сетка модели авторами не приводится.

Материал образца с коэффициентом Пуассона $\mu=0,3$ считается упругопластическим с линейным упрочнением. Секущий модуль $E^{\prime}$, определяющий степень упрочнения варьировался в процессе расчетов и задавался в долях модуля Юнга, принятого равным $E=200$ ГПа. Предел текучести полагался равным $\sigma_{T}=350 \mathrm{MПа.}$

Полученные авторами результаты расчётов показывают, что моделирование процесса образования шейки возможно без введения каких-либо предположений о наличии начальных несовершенствах в геометрии образцов. Это достигается применением уравнений геометрически нелинейной теории упругости и пластичности

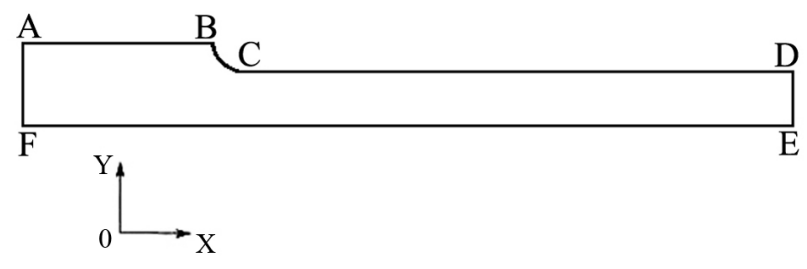

Рис. 1. Геометрия расчётной модели Д.В. Бережнова и В.Н. Паймушина [6].

Fig. 1. The geometry of the computational model D. V. Berezhnova and V.N. Paimushina [6]. 
при конечных деформациях и перемещениях, физических зависимостей, связывающих между собой истинные напряжения и деформации, построенных при учете упругопластичности материала, а также корректного описания геометрии головки образца и способа их нагружения.

На рис. 2 представлен процесс локализации шейки при моделировании авторами процесса растяжения образца с $E^{\prime}=1 \times 10^{-6} E$ на различных этапах удлинения от 84 мкм до 1680 мкм.

На рис. 3 представлены иллюстрации локализаций шейки в расчёте при секущих модулях составляющих $1 \times 10^{-6} \div 0,96$ Е при удлинении 1680 мкм.

Авторы отмечают, что использованные физические зависимости в виде уравнений Прандтля-Рейсса являются простейшими и позволяют корректно описать процесс формирования шейки лишь на начальном этапе её зарождения и развития.

\section{3. Моделирование процесса обра- зования шейки в коде ANSYS}

В работе [6] при моделировании использовались меры истинных деформаций удлинений єі и сдвигов $\sin \gamma \mathrm{ij}$ по В.В. Новожилову. При учёте больших деформаций в комплексе ANSYS используется мера деформаций Генки:

$$
[\varepsilon]=\ln [U]
$$

где $[U]$ - матрица искажения формы тела (матрица удлинений) [8]. В качестве физического закона пластичности в ANSYS используется пластическая теория течения с критерием начала пластичности Мизеса.

Представляет интерес воспроизведение расчёта по работе [6] в верифицированном [8], [9] расчётном комплексе.

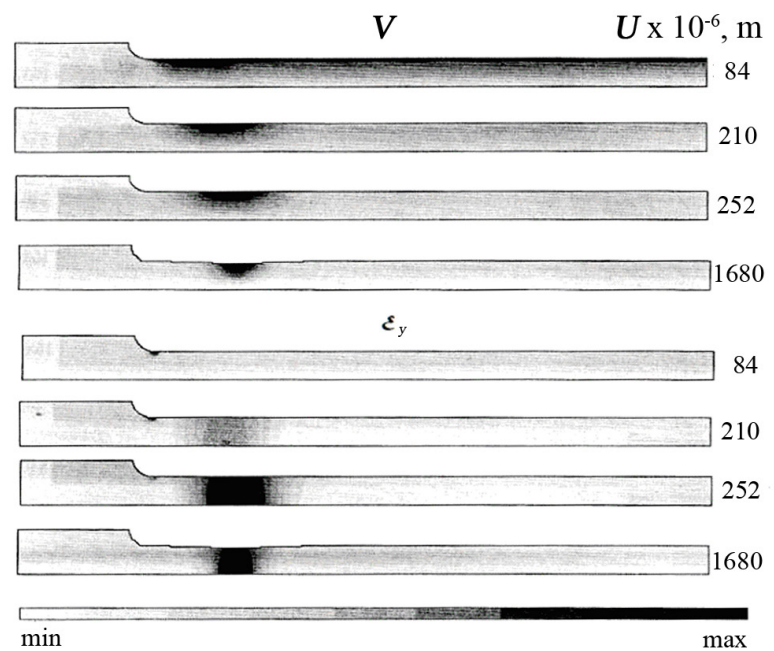

Рис. 2. Процесс локализации шейки на различных этапах растяжения в [6] при секущем модуле $E^{\prime}=1 \times 10^{-6} E$, где $V$ и $\varepsilon_{y}$ поперечные перемещения и деформации соответственно.

Fig. 2. Visualization of necking at different stages of tension in [6] with tangent modulus $E^{\prime}=1 \times 10^{-6} E$, where $V$ and $\varepsilon_{y}$ are transverse displacement and strain respectively.
Постановка задачи эквивалентна постановке в [6] по геометрическим размерам, способам приложения нагрузки и механическим характеристикам материалов. Расчёты выполнены с учётом физической (упругопластические свойства) и геометрической (большие деформации) нелинейностей. Расчёт производился в осесимметричной постановке. Модель содержала 7520 четырёхугольных элементов второго порядка (с квадратичной функцией формы) и 23365 узлов. Характерный размер элемента в зоне головки образца составлял 1 мм, в зоне рабочей части образца - 0,5 мм, дуга галтели была разбита на 32 элемента. Геометрия модели и пример сетки в области галтели приведены на рис. 4.

Расчёт проводился до конечного удлинения 1680 мкм с начальным инкрементом удлинения в 33,6 мкм и автоматической коррекцией инкремента средствами программного комплекса на основании скорости сходимости.

На рис. 5 представлен процесс локализации шейки при моделировании процесса растяжения образца с $E^{\prime}=1 \times 10^{-6} E$ на различных этапах удлинения от 102 мкм до 1680 мкм.

На рис. 6 представлены иллюстрации локализаций шейки в расчёте при секущих модулях составляющих $1 \times 10^{-6} \div 0,9$ Е при удлинении 1680 мкм.

Картины распределения показывают, что результаты, полученные при учете больших деформаций в ANSYS, качественно совпадают с результатами работы [6]. Однако количественно полученные данные сильно разнятся. Более чем на порядок отличаются величины секущих мо-

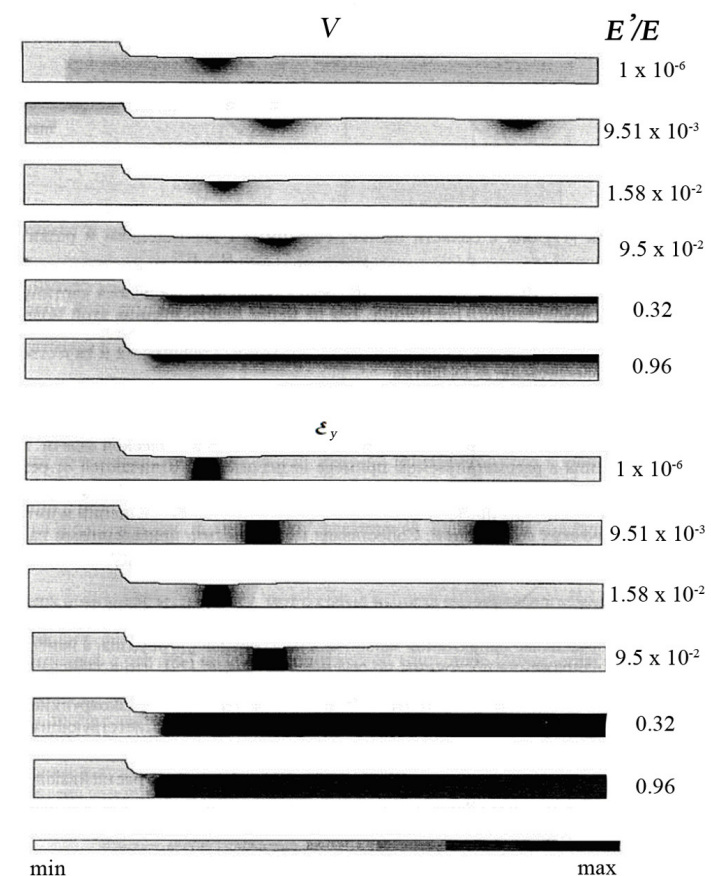

Рис. 3. Локализация шейки при расчёте в зависимости от секущего модуля в [6], где $V$ и $\varepsilon_{y}$ - поперечные перемещения и деформации соответственно.

Fig. 3. Neck location changing in simulations with different tangent modulus in [6], where $V$ and $\varepsilon_{y}$ are transverse displacement and strain respectively. 
дулей для одинаковых картин напряженно-деформированного состояния в том и в другом случае. Кроме того, зависимость положения локализации шейки от величины секущего модуля в [6] (рис. 3) носит сложный характер с образованием в ряде случаев нескольких шеек, а также скачками положения шейки при переходе от предыдущего значения секущего модуля к следующему. В то же время в результатах расчёта в коде ANSYS аналогичная зависимость имеет характер непрерывного распределения с локализацией шейки около галтели при практически идеально-пластической характеристике материала, постепенном смещении области локализации к центру

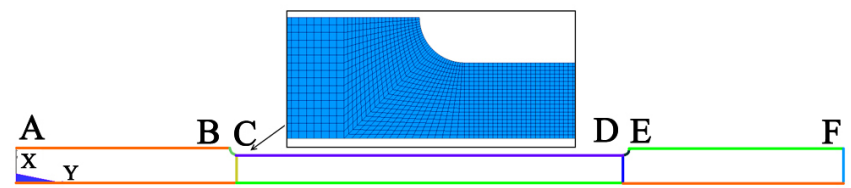

Рис. 4. Геометрическая модель и сетка.

Fig. 4. Geometric model and mesh.
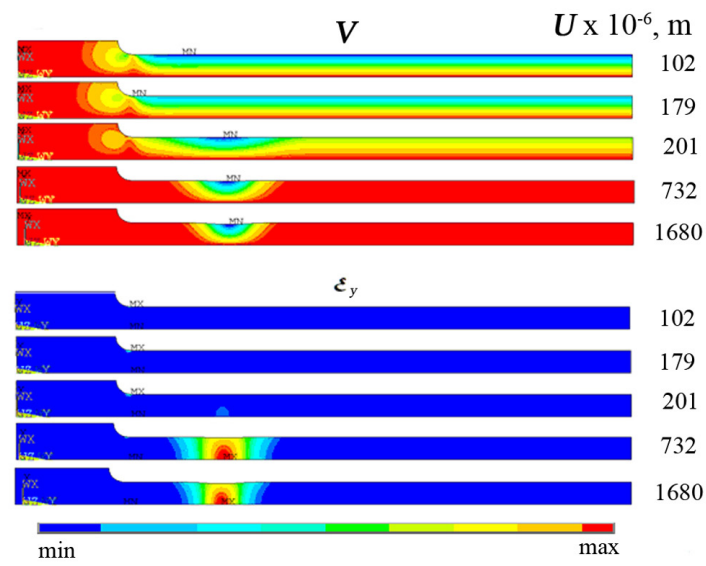

Рис. 5. Процесс локализации шейки на различных этапах растяжения при расчёте в ANSYS, где $V$ и $\varepsilon_{y}-$ поперечные перемещения и деформации соответственно.

Fig. 5. Visualization of necking at different stages of tension in ANSYS simulation, where $V$ and $\varepsilon_{y}$ are transverse displacement and strain respectively.

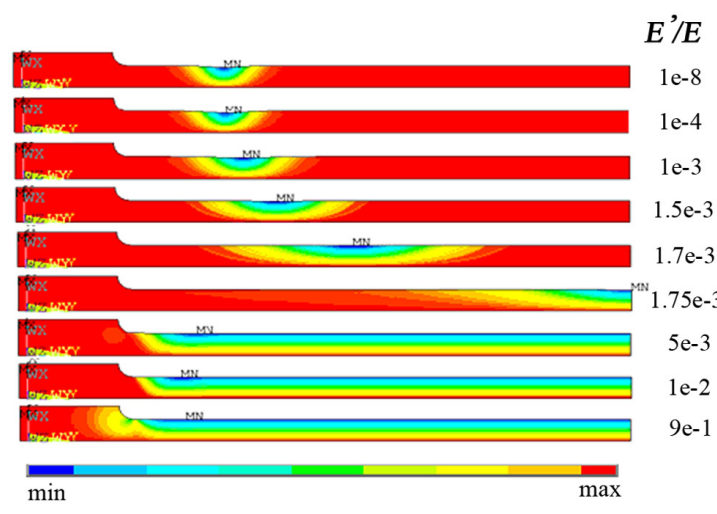

Рис. 6. Локализация шейки при расчёте в зависимости от секущего модуля при расчёте в ANSYS.

Fig. 6. Neck location changing in ANSYS simulations with different tangent modulus. образца к величине секущего модуля $1,75 \times 10^{-3} E$ и вырождении локализации как таковой в практически равномерную по длине деформацию при величине секущего модуля превышающей $1,9 \times 10^{-3} \mathrm{E}$.

Анализ такого рода расхождений в результатах затруднён, так как коммерческий исходный код ANSYS недоступен для анализа и в то же время авторами [6] не приведён собственный исходный код для анализа. Отметим только, что в верификационных матрицах ANSYS [8], [9] присутствуют задачи, решённые с учётом больших деформаций.

\section{4. Расчётно-экспериментальный анализ локализации шейки}

Дальнейшее исследование закономерностей локализации шейки проводилось на основе экспериментальных результатов испытаний цилиндрических образцов на растяжение.

Для испытаний были изготовлены образцы различных типоразмеров, по 5 штук каждого типоразмера. Образцы типоразмера 1 изготавливались в соответствии с ГОСТ 1497 - 84. Длина рабочей части образцов типоразмера 1 составляла 80 мм, диаметр 8 мм. Образцы типоразмера 2 изготавливались удлинёнными в два раза по сравнению с образцами типоразмера 1 для отслеживания динамики изменения положения шейки при изменении размерных соотношений. Длина рабочей части этих образцов составляла 160 мм, диаметр - 8 мм. Образцы обоих типоразмеров изготавливались из двух различных материалов - конструкционной стали Ст.20 и нержавеющей стали 08X18H12T (321 AISI USA). Coотношение диаметра к длине рабочей части образца образцов типоразмера 2 аналогично соотношению, применяющемуся в модели авторами [6] Для снижения влияния способа обработки и локальных дефектов шероховатость поверхностей при изготовлении обеспечивалась $R_{a}=0,63$ мкм.

Испытания проводились на разрывной машине INSTRON 5982 с диапазоном силоизмерителя 100 кН. Процесс деформирования двух образцов из выборки также был зарегистрирован методом цифровой корреляции изображений (ЦКИ) посредством оптической системы Vic-3D RT Correlated Solutions [10]. Деформации этих образцов зарегистрированы в процессе растяжения внешним тензометрическим датчиком.

После разрыва образцов производилось измерение расстояния от края ближайшего захвата до минимального диаметра в шейке (место локализации шейки).

Для проверки выдвинутой авторами [6] гипотезы о воспроизведении в расчёте экспериментального места локализации шейки было проведено моделирование в коде ANSYS.

Численное моделирование проведено для образцов всех типоразмеров. В качестве исходных данных использовались точные геометрические размеры каждого образца и истинная диаграмма деформирования. Вычисления производились в осесимметричной постановке, расчётная модель представляла собой $1 / 2$ продольного сечения образца. 
Граничные условия модели представляли собой предписанные нулевые перемещения на участке АВ (см. рис. 4) и предписанное ненулевое перемещение на участке EF. Перемещение выбиралось равным перемещению захвата при испытании соответствующего образца.

Пересчёт напряжений и деформаций в истинные величины до временного сопротивления производился по известным формулам:

$$
\begin{gathered}
S=P / F_{0} \cdot(1+\varepsilon), \\
e=\operatorname{Ln}(1+\varepsilon),
\end{gathered}
$$

где $S$ - истинные напряжения, $P$ - усилие, снятое силоизмерителем машины, $F_{0}-$ начальная площадь поперечного сечения образца, $e-$ истинная деформация, $\varepsilon-$ инженерная деформация, снятая с накладного тензометра.

Последний участок диаграммы достраивался соединением крайней точки, полученной по формулам (3) и истинной точки разрыва, координаты которой определены как:

$$
\begin{gathered}
S_{k}=P_{k} / F_{k}, \\
e_{k}=\psi / 1-\psi+S_{k} / E,
\end{gathered}
$$

где $P_{k}$ - значение нагрузки в момент разрыва, $F_{k}-$ площадь поперечного сечения после разрыва, $e_{k}-$ максимальная суммарная деформация образца, $\psi$ - относительное сужение после разрыва образца.

Отметим, что построенная таким образом диаграмма не является диаграммой, описывающей истинную зависимость между напряжениями и деформациями при глубоком пластическом деформировании в материале, поскольку опирается на предположение о равномерности распределения напряжений по минимальному поперечному сечению шейки в момент перед разрывом. Известно [4], что это распределение является существенно нелинейным для всех компонент.

Пример восстановленной таким образом диаграммы деформирования для образца типоразмера 1, изготовленного из конструкционной стали, представлен на рис. 7.

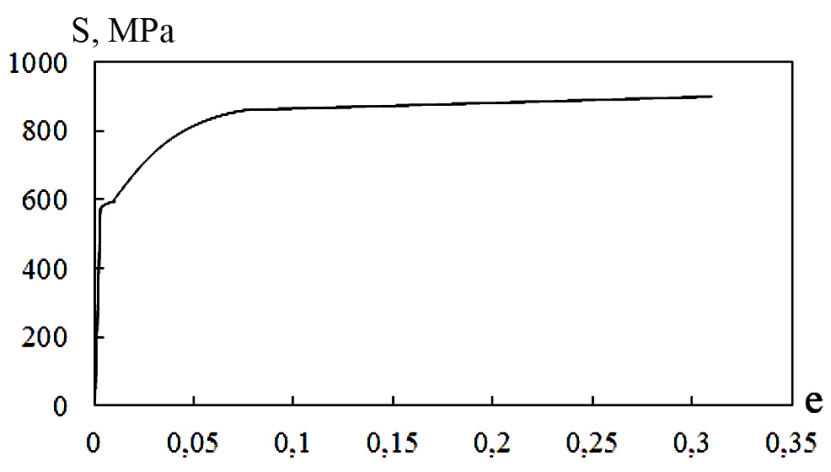

Рис. 7. Диаграмма деформирования образца в истинных величинах.

Fig. 7. Stress-strain diagram of the specimen in the true values.

\section{5. Результаты исследования}

В результате испытаний на растяжение установлено, что в образцах одного типоразмера, выполненных из одного материала, положение локализации шейки имеет достаточно слабый разброс. Так, в образцах типоразмера 1, изготовленных из конструкционной стали, усреднённое место локализации шейки находится на расстоянии 13,4 1,5 мм от края ближайшего захвата. Максимальное и минимальное зафиксированные значения при этом составляют 15 мм и 12 мм соответственно.

Это лишний раз подтверждает гипотезу о том, что в образцах одного типоразмера, из одного материала, не имеющих значимых геометрических несовершенств и испытанных по одинаковой схеме, шейка локализуется в одном и том же месте. Это свидетельствует в пользу того, что положение шейки определяется не положением микроскопических несовершенств образца, а геометрическими и механическими свойствами образца и схемой испытания.

На рисунках 8-11 приведено сопоставление картин распределения радиальных перемещений в расчётах с фотографиями соответствующих реальных образцов после испытания.

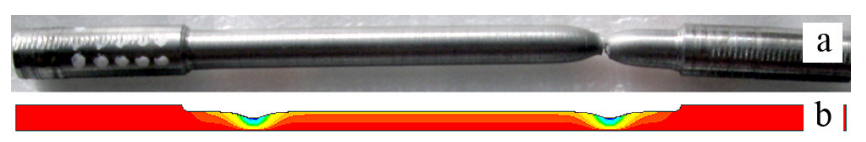

Рис. 8. Образец типоразмера 1 из конструкционной стали. Фотография разрушенного образца - (a), расчетная картина радиальных деформаций - (b).

Fig. 8. Structural steel specimen with size type 1. Foto of destroyed specimen - (a), radial displacement field from simulation - (b).

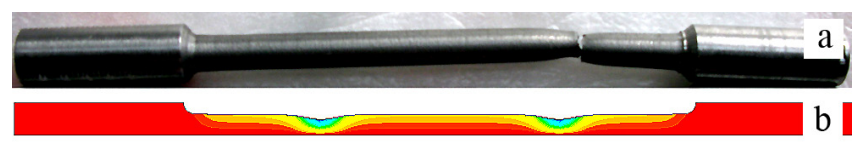

Рис. 9. Образец типоразмера 1 из нержавеющей стали. Фотография разрушенного образца - (a), расчетная картина радиальных деформаций - (b).

Fig. 9. Stainless steel specimen with size type 1. Foto of destroyed specimen - (a), radial displacement field from simulation - (b).

Рис. 10. Образец типоразмера 2 из конструкционной стали. Фотография разрушенного образца - (a), расчетная картина радиальных деформаций - (b).

Fig. 10. Structural steel specimen with size type 2. Foto of destroyed specimen - (a), radial displacement field from simulation - (b).

Рис. 11. Образец типоразмера 2 из нержавеющей стали. Фотография разрушенного образца - (a), расчетная картина радиальных деформаций - (b).

Fig. 11. Stainless steel specimen with size type 2. Foto of destroyed specimen - (a), radial displacement field from simulation - (b). 
Сопоставление результатов расчётного определения места локализации шейки с усредненными экспериментальными значениями по 5 образцам приведено в табл. 1.

В ряде расчётов было отмечено формирование двух взаимно симметричных шеек в одном образце. Анализ записей, сделанных методом ЦКИ для двух образцов, позволяет установить, что в процессе растяжения реального образца на начальном этапе, деформации распределяются по образцу равномерно, затем формируются две взаимно симметричные области концентрации деформаций, после чего, незадолго до достижения величины временного сопротивления, одна из областей расформировывается, а на месте оставшейся формируется шейка (рис. 12).

табл. 1. Сопоставление положений шейки, полученных в расчёте и эксперименте.

Table 1. Experimental and simulated neck location comparison.

\begin{tabular}{|c|c|c|c|}
\hline $\begin{array}{c}\text { Образец } \\
\text { (тип, материал) } \\
\text { Sample } \\
\text { (size, material) }\end{array}$ & $\begin{array}{c}\text { Расчет, } \\
\text { мм } \\
\text { Calculation, } \\
\text { mm }\end{array}$ & $\begin{array}{c}\text { Эксперимент, } \\
\text { мм } \\
\text { Experiment, } \\
\text { mm }\end{array}$ & $\begin{array}{c}\text { Разница, } \\
\% \\
\text { Difference, } \\
\%\end{array}$ \\
\hline $\begin{array}{c}\text { Тип 1 } \\
\text { Сталь Ст.20 } \\
\text { Туре 1 } \\
\text { Steel St.20 }\end{array}$ & 11,4 & 13,4 & 15,0 \\
\hline $\begin{array}{c}\text { Тип 1 } \\
\text { Сталь 321 AISI } \\
\text { Туре 1 } \\
\text { Steel 321 AISI }\end{array}$ & 20,3 & 25,0 & 19,0 \\
\hline $\begin{array}{c}\text { Тип 2 } \\
\text { Сталь Cт.20 } \\
\text { Туре 2 } \\
\text { Steel St.20 }\end{array}$ & 12,5 & 15,0 & 17,0 \\
\hline $\begin{array}{c}\text { Тип 2 } \\
\text { Сталь 321 AISI } \\
\text { Туре 2 } \\
\text { Steel 321 AISI }\end{array}$ & 19,9 & 21,8 & \\
\hline
\end{tabular}
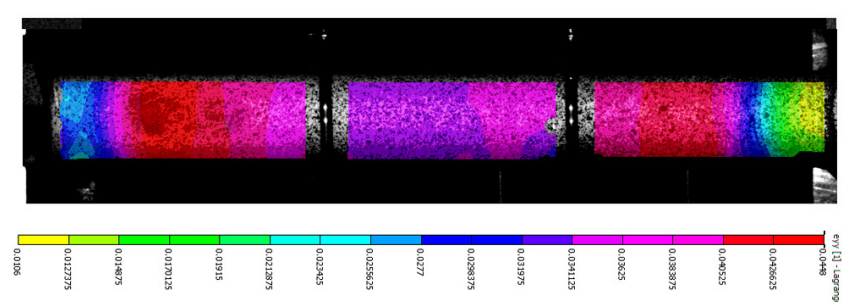

Puc. 12. (Color online) Цветная картина поля поперечных деформаций, зарегистрированная методом цифровой корреляции изображений образца типоразмера 1 из нержавеющей стали.

Fig. 12. (Color online) Color field pattern of transverse deformations registered on size type 1 stainless steel specimen by digital image correlation.

\section{6. Выводы}

Таким образом, по результатам выполненного исследования можно сделать следующие выводы.

При МКЭ-моделировании процесса растяжения цилиндрического образца с учётом упругопластических свойств материала и больших перемещений в расчёте наблюдается образование шейки. Подобный эффект наблюдается как в расчётном коде, использованном в [6], так и в коммерческом коде ANSYS.

При испытании цилиндрических образцов на растяжение у образцов одного типоразмера, выполненных из одного материала и не содержащих значительных геометрических несовершенств, наблюдается образование шейки преимущественно в одном и том же месте образцов.

При сопоставлении экспериментальных результатов с расчётами можно отметить качественное совпадение. Изменение места положения шейки при изменении типоразмера и материала образца в соответствующих расчётах и испытаниях происходит в одинаковом направлении. Однако отличие расчётных положений шейки на 9 - 19\% не позволяют говорить о количественном совпадении результатов.

Благодарность/Acknowledgement. Статья написана в рамках программы по повышению конкурентоспособности НИЯУ «МИФИ».

\section{Литература/References}

1. G.D. Del, S.S. Oding. Prikladnaya mekhanika. 11(18), 86-91 (1982). (in Russian) [Г.Д. Дель, С. С. Одинг. Прикладная механика. 11(18), 86-91 (1982)].

2. J.B. Friedman. Mechanical properties of metals/In two parts. Part 2. Mechanical tests. Structural strength. M., Mechanical Engineering. 1974. 369. (in Russian) [Я.Б. Фридман. Механическиесвойстваметаллов/Вдвухчастях. Часть 2. Механические испытания. Конструкционная прочность. М., Машиностроение. 1974. 369с.]

3. N.N. Davidenkov, N.I. Spiridonova. Zavodskaya laboratoriya. 6, $583-593$ (1945).

4. A.M. Zhukov. Engineering collection. 2(5), 34-51 (1949). (in Russian) [A. М. Жуков. Инженерный сборник. 2(5), 34 - 51 (1949)].

5. V. G. Kuteikin. Zavodskaya laboratoriya. 9, 53- 55 (2002).

6. D. V. Berezhnoi, V.N. Paimushin. Prikladnaya matematika i mekhanika. 4, 635-659 (2011).

7. V. V. Novozhilov. The theory of elasticity. L., Sudpromgiz. 1958. 370 p. (in Russian) [В. В. Новожилов. Теория упругости. Л., Судпромгиз. 1958. 370 с.]

8. Release 16.2 Documentation for ANSYS [electronic document]/ANSYS Inc. Electronic data and software (104019 files: 10660130531 bytes).

9. NAFEMS search engineering analysis and simulation FEA, Finite Element Analysis, CFD, Computational Fluid Dynamics, and Simulation. NAFEMS Ltd., Hamilton, United Kingdom. (2016).

10. M. A. Sutton, J. J. Orteu, H. W. Schreier. Image Correlation for Shape, Motion and Deformation Measurements: basic concepts, theory and applications. Springer. P.321. (2009). 